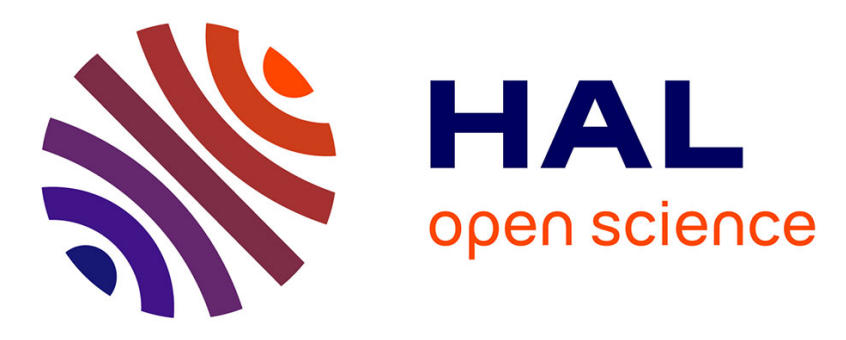

\title{
Design of a decision support system for buried pipeline corrosion assessment
}

Laurence Boudet, Jean-Philippe Poli, Alicia Bel, François Castillon, Frédéric Gaigne, Olivier Casula

\section{- To cite this version:}

Laurence Boudet, Jean-Philippe Poli, Alicia Bel, François Castillon, Frédéric Gaigne, et al.. Design of a decision support system for buried pipeline corrosion assessment. 17th International Conference on Information Processing and Management of Uncertainty in Knowledge-Based Systems. Applications, Jun 2018, Cadiz, Spain. pp.74-85, 10.1007/978-3-319-91479-4_7 . cea-01838410

\section{HAL Id: cea-01838410 https://hal-cea.archives-ouvertes.fr/cea-01838410}

Submitted on 26 Feb 2022

HAL is a multi-disciplinary open access archive for the deposit and dissemination of scientific research documents, whether they are published or not. The documents may come from teaching and research institutions in France or abroad, or from public or private research centers.
L'archive ouverte pluridisciplinaire HAL, est destinée au dépôt et à la diffusion de documents scientifiques de niveau recherche, publiés ou non, émanant des établissements d'enseignement et de recherche français ou étrangers, des laboratoires publics ou privés. 


\title{
Design of a Decision Support System for Buried Pipeline Corrosion Assessment
}

\author{
Laurence Boudet \\ CEA, LIST, Data Analysis and System Intelligence Laboratory, \\ 91191 Gif-sur-Yvette cedex, France. \\ Jean-Philippe Poli \\ CEA, LIST, Data Analysis and System Intelligence Laboratory, \\ 91191 Gif-sur-Yvette cedex, France. \\ Alicia Bel \\ CEA Tech Aquitaine, 33600 Pessac, France \\ François Castillon \\ Frédéric Gaigne \\ TIGF, 64000 Pau, France \\ TIGF, 64000 Pau, France \\ Olivier Casula \\ CEA Tech Aquitaine, 33600 Pessac, France
}

February 25, 2022

\begin{abstract}
Maintaining the level of integrity of pipeline networks to guarantee at least a reliable and safe service is a challenge operators of such networks are facing everyday. TIGF is one of the French operator which manages $5000 \mathrm{~km}$ of pipelines in the south-west quarter of France. This paper presents the work from our collaboration. It results in a decision-making tool which automatically ranks the pipeline sections regarding the risk of deterioration (damages and corrosion) and the gravity of the consequences, indicating which pipeline sections should be excavated. The tool relies on a fuzzy expert system which gathers 26 input variables, processes more than 300 rules, classifies the risk of deterioration into 7 classes and estimates the gravity. The rules are a formalization of human experts: the fuzzy logic helps to tackle the vagueness of their knowledge and the measurement inaccuracy of some of the 26 input variables. The method has been tested on past excavations to assess its performances.

Keywords: Corrosion, pipeline networks, risk assessment, decision-making, fuzzy expert system
\end{abstract}




\section{Introduction}

Everywhere in the world, high-pressure pipelines are used to transport gaz from the production and storage sites to the customers. The major challenge with such networks of pipelines is to maintain their level of integrity to guarantee at least a reliable and safe service. Regarding the conditions of the pipelines (ground, buried, subsea etc.) and their environment (soil, coating etc.), different kinds of corrosion may damage them $[6,8,14]$. The phenomenon is too complex and too little understood to be modeled analytically, or only in specific context (coating material, soil type and features, pipe age, ...).

However, different measures can help to identify which pipeline sections are affected by a certain type of corrosion. We can distinguish several approaches to exploit these measures. Bayesian techniques have been used to incorporate uncertainty and measurement errors $[4,5,14]$ and to either compute the probabilities of corrosion occurrence and consequences or to assess the size of the corrosion. Other papers introduce a probabilization of physical models like [9] which predicts the corrosion remaining life. All those methods are also related to Monte-Carlo simulation to overcome the lack of data and suffer from the difficulty to choose the most proper distributions. Another way of taking the uncertainty and inaccuracy into account is performed by fuzzy logic $[11,10,15]$ : various qualitative and quantitative factors are considered in assessing the security of pipeline network. The authors fuzzify classical models used in risk assessment. We can also cite [1] in which the authors introduce an expert system to suggest the adequate coating regarding several quantitative and qualitative parameters. It ranks all the available materials and the most suitable one is chosen. In a more anecdotal way, other papers apply Multi-Attribute Utility Theory (MAUT) [3] and machine learning [7] to risk assessment.

In this article, we present an application of fuzzy expert system to tackle both the recognition of the type of deterioration and the risk assessment. Fuzzy expert systems can handle a cold start, i.e. the lack of data at the start of the project, knowledge vagueness and measurement uncertainty. Moreover, as corrosion depends on environmental conditions, the decision making tool can be easily adapted from a region to another.

We focus on the network managed by our partner, TIGF, responsible of the pipeline network in the south-west quarter of France. The whole network is buried and TIGF is facing corrosion of different natures. Ground inspection must help them to ensure a section of the network is affected by corrosion. The difficulty resides in the fact that several criteria have to be merged to make the decision. Our approach is based on knowledge modeling and testing on data.

The paper is structured as follows. The next section introduces the context of corrosion of non-piggable pipelines. Then, section 3 motivates the choices made for the decision support system (DSS) which has been built for TIGF in order to assess risk and corrosion types. Section 4 describes the knowledge modeling of human experts with fuzzy logic and introduces the user interface of the system. The results of the application of the tool are presented in section 5. Finally, we draw a conclusion and some perspectives to this work. 


\section{The case-study}

Pipeline pigging is an effective way to accurately locate steel defects and metal loss. Unfortunately, this method is inappropriate for some pipe configurations, such as small diameter or multi-diameter pipes. Therefore, above ground inspection surveys are conducted to gather information about the whole network condition. TIGF mainly uses the Direct Current Voltage Gradient (DCVG) technique.

We explain in this paragraph the principle of the DCVG technique. If the pipe is exposed at holidays in its protective coating, the current impressed by the cathodic protection system will flow from the soil into the bare steel. It results in voltage gradients in the soil surrounding the defect. The DCVG method consists in pulsing the input current signal and detecting associated voltage gradients in the soil above the pipeline, that betray the presence of a soil-metal interface. To this end, an operator performs regular measures with a milli-voltmeter of the voltage drop between two electrodes placed on the soil surface at a distance that remains constant (about 1.5 meters). As the operator approaches a coating defect, he observes an increasing pulsing signal. This signal finally stabilizes then decreases as the defect is passed.

Each defect severity is characterized by its value of \%IR, which is computed from DCVG measures. Then, thanks to calibrated references, the size of the steel surface exposed can be estimated.

DCVG surveys return alerts on the pipeline protective coating. However, it does not inform about the cathodic protection state, nor does it imply a real metal deterioration. To better assess the risk of a pipeline defect, TIGF gathers additional data:

- the pipeline specific features (age, type of coating,...);

- the pipeline environment (presence of stray currents, soil resistivity, soil bacteria,...);

- the history of the pipeline cathodic protection.

All of this information is carefully examined with multiple risks in mind. In addition to mechanical attacks and high-voltage damages, a typology of 5 different types of corrosion is considered (see figure 1): stray current corrosion, corrosion caused by alternating current (AC corrosion), corrosion under a disbonded shielding coating, bacterial corrosion and insufficient cathodic protection. Finally, the gravity of the consequences that a severe metal defect would have is a crucial parameter that is also carefully taken into consideration. A pivotal factor is the pipeline proximity from any public location or infrastructure.

Conducting a systematic analysis of the thousands of coating defects that are detected each year by DCVG surveys is a real challenge. Indeed, since the excavation of a pipeline section is very expensive, only few anticipated defects can be checked. Thus, it requires to apply a wide expertise in a consistent fashion to a large variety of configurations. In this context, a decision support tool 

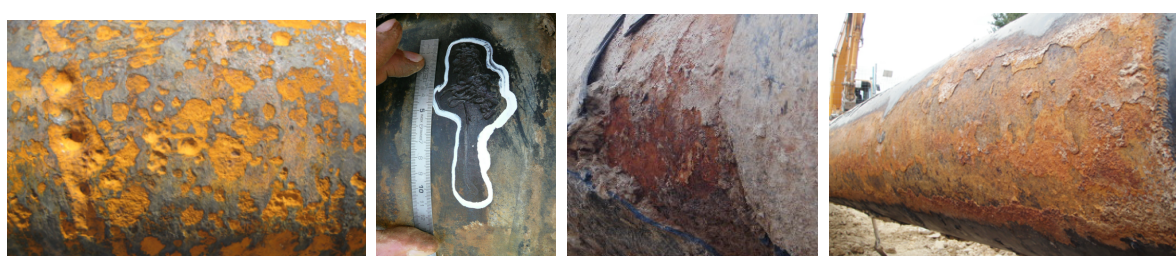

Figure 1: Example of different corrosion shapes and surfaces.

offers clear benefits.

\section{Decision support system design}

\subsection{Technology choice}

The proposed DSS assists human experts in identifying the most critical coating defects for excavation. There are quite a lot of available measurements or information (26 variables per coating defect): some of them are qualitative, others are quantitative. There are a lot of individuals (several thousands of defects) in the first data set but very few of them were labeled at the beginning of the study (around 50 for one DCVG survey). There are 7 risks of metal deterioration. This data set is unbalanced since one deterioration type represents two thirds of actual defects and some are missing. Moreover, each defect should not be considered independent from the others because a set of defects (even a lot of them sometimes) can be detected at different locations of the same pipeline. Their features are then correlated. Thus, it was not possible to learn the features of the defects because of the small number of labeled individuals available at the beginning of the study nor to apply clustering techniques because of the difficulty to find a distance dealing with both quantitative and qualitative features and separating pipeline sections with or without deterioration.

Despite these observations, the DSS should be usable immediately because it is not conceivable to wait a series of yearly acquisition campaigns. Thus, it has to exploit the expert background and experience at TIGF. It will allow to formalize and to structure this knowledge on the one hand, and to treat all the coating defects in an homogeneous and systematic way on the other hand. Unfortunately, underground corrosion phenomena comprehension is much more a matter of vague and uncertain knowledge and understanding than a precise and definitive knowledge. Indeed, there are multiple factors implied in corrosion formation and it is a local phenomenon because it depends on soil features. Moreover, coating used for pipes as well as the type of protections changed over the last 70 years because of acquired experience on coating ageing and corrosion and improvements of materials.

Obviously, the DSS should improve the effectiveness of realized excavations compared to the ones decided without it. Moreover, a higher success rate will 
allow TIGF to reduce the number of excavations following DCVG surveys.

Taking into account these different points, we proposed to use a fuzzy expert system to model expert knowledge. It may be designed from expert interviews before the availability of a data set. Fuzziness is helpful to deal with vagueness and uncertainty. Two other features pleaded for a fuzzy expert system. Firstly, the DSS is not a black box: its suggestions are justified thank to activated rules and can be understood by the user. Secondly, if we found out that modeled knowledge is imprecise or incorrect, it can be updated by only changing associated fuzzy rules. These ones are saved in external files and automatically loaded at the next start of the DSS. Thus, expert knowledge can be refined with new experiences and the virtuous circle of knowledge improvement is possible.

\subsection{DSS life cycle}

There are five steps in the design and exploitation of the DSS:

1. System design: the DSS is designed with human experts at TIGF. Knowledge is gathered and modeled with fuzzy rules. A dedicated graphic user interface (GUI) is specified and implemented. During this phase, we decided to use some features that were available in the databases but not extracted for this purpose yet. Then, the first annual DCVG survey with actual values was formatted more or less automatically to be used by the DSS.

2. Manual corrections: The DSS results are evaluated and analyzed with the first data set. Some rule improvements are identified by comparing the actual values of the defects to what was modeled by knowledge elicitation. Then, more data are collected and formatted during this step. Several annual DCVG surveys are automatically extracted. There are 330 labeled metal defects in this second data set (including the first one). Knowledge update is natural when using fuzzy rules, and is very easy by using the GUI [12] of our fuzzy expert system. We followed two ways to discover corrections to apply. Firstly, parameters used for defining fuzzy sets of some variables are refined by a statistical analysis when sufficient data are available. Secondly, this data set enables to question about the influence of some parameters on some risks.

3. First exploitation: The DSS is used to evaluate the excavations to select for the next yearly DCVG survey. The software is used to navigate between coating defects, visualize, analyze and understand possible risks. Moreover, reports are automatically generated on most possible risks.

4. Automatic improvements: Data registered in case of swabbing of pipelines at accessible location (in particular, at manholes) are automatically extracted with their actual values. The latter are much more numerous than the labeled ones obtained by DCVG survey (several thousands). 
Optimization of fuzzy rules is then possible with the second and the third data sets for the most frequent observed risks. After validation by human experts at TIGF, correction of fuzzy sets are made with the dedicated GUI [12]. This step is not introduced in this article.

5. Full exploitation: After fine tuning of fuzzy rules by optimization, the DSS is used to choose the defects to excavate for the next year. Efficiency and usefulness of the DSS can be assessed by different considerations: success rate of realized excavations, spent time to select defects to be excavated, justification of decisions and evolution of repair costs. The latter depend on the number of defects selected for excavation which will decrease in several years if the success rate raises.

\section{Knowledge modeling and visual tool}

\subsection{Human expert knowledge modeling}

The DSS evaluates the criticity of each coating defect in a large variety of configurations in a structured and systematic way. The work conducted to make the knowledge of human experts fully explicit. First of all, we identified nine indicators including seven risks of deterioration which must be distinguished. Some works close to the pipe may simply damage it if there is no mechanical protection. Another pipe damage may arise because of a close high-voltage line. Moreover, five corrosion types are considered: stray current corrosion, corrosion caused by alternating current, corrosion under a disbonded shielding coating, bacterial corrosion and insufficient cathodic protection. The development of each corrosion type is multi-factorial: it depends on intrinsic factors of the pipe (age, type of coating, ...) and extrinsic factors given by environmental features (presence of stray currents, soil resistivity, soil bacteria,...). The \%IR based on DCVG measure gives an information about the estimated surface of the coating defect of the pipe. The estimated risks are considered with a factor that indicates a higher risk of deterioration based on background (history of the pipeline) and a factor that assesses the severity of a potential metal defect (its proximity from any public location or infrastructure). All this information is available in knowledge management systems at TIGF in different forms: either quantitative, categorical or boolean. Only one part of this information was already used to assess the risk of each coating defect. In some cases, only the simplest raw information was used such as the presence of bacteria in the soil while a concentration was known.

After having identified the different inputs and outputs of the DSS, we have to understand the influence of each input on the outputs. During the interviews, a lot of expressed knowledge was in the form "the higher the value of $\mathrm{X}$, the higher the risk of A" like in the rule "the higher the age of the pipe, the higher the risk of the corrosion of type A", or at the contrary the form "the lesser the resistivity, the higher the risk of the corrosion of type A". We could have used 


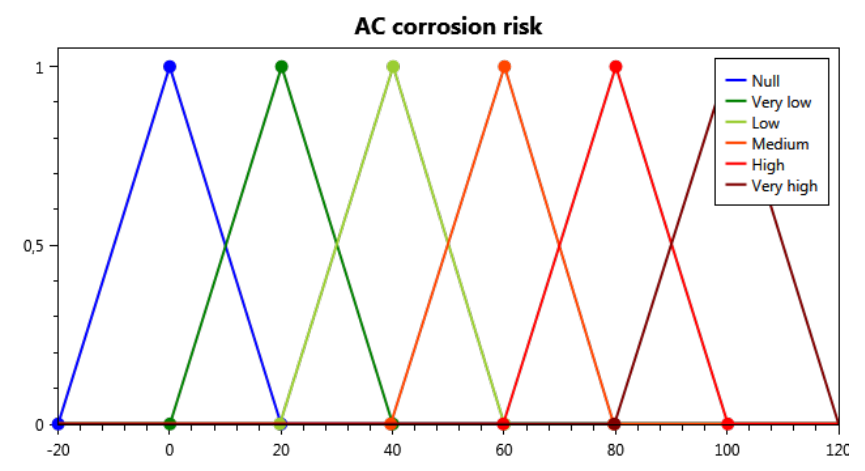

Figure 2: Fuzzy sets for risk level, in this case for alternating current corrosion

powerful and synthetic fuzzy rules like fuzzy gradual rules or those based on "all the more" clauses [2] for instance. However, there were generally several factors that influence each risk and we did not know which relation between the inputs and the outputs should be chosen. Thus, we decided to adopt a grid structure for modeling the input - output relations for each module of fuzzy rules. Indeed, it is very easy to understand for the corrosion domain experts who were not used to fuzzy logic. Moreover, it is simple to update a conclusion according to one (or several) influencing parameter(s). However, we did not follow a strict flat grid structure approach. When possible, intermediate variables were introduced in order to synthesize pieces of information at intermediate level. The latter ones are used into other modules of fuzzy rules thanks to chaining. This provides a hierarchical structure of the fuzzy system [13] that makes it more concise and understandable. It is used for instance for establishing a bacterial risk due to the soil characteristics. This risk depends on 4 input variables of pipe environment. Then, the bacterial risk is considered with the coating material to determine the bacterial corrosion risk of the pipe.

Thus, we modeled each variable (either input or output) by a linguistic variable and built fuzzy rules to make the expert knowledge more explicit. We chose to model each risk of pipe deterioration with the same set of fuzzy sets (see figure 2): the risks come from null to very high with 4 intermediate levels. The risk suggested by the pipeline history is rated with the same levels. With the Mamdani inference system, the numerical defuzzified value of each risk belongs to $[0,100]$. A boolean alert about the history is given by coupling the potential corrosion risk with the one implied in the history of the pipe close to the coating defect when known. Indeed, an old mechanical attack does not tell anything on a possible corrosion due a bacteria for instance. Finally, the severity of the consequences is evaluated with four fuzzy terms from low to critical.

The table 1 shows the risk level of one type of corrosion according to four input variables for one specific coating. There are 24 rules for this example, which is a minimum because we used only 2 terms for each parameter. It represents a gradual influence of each parameter on the risk level. The worst case occurs 


\begin{tabular}{|c|c|c|c|c|}
\hline $\begin{array}{c}\text { Level of } \\
\text { risk }\end{array}$ & $\begin{array}{c}\text { Diameter is S } \\
\text { \& age is low }\end{array}$ & $\begin{array}{c}\text { Diameter is S } \\
\text { \& age is high }\end{array}$ & $\begin{array}{c}\text { Diameter is L } \\
\text { \& age is low }\end{array}$ & $\begin{array}{c}\text { Diameter is L } \\
\text { \& age is high }\end{array}$ \\
\hline $\begin{array}{c}\text { Resistivity is H } \\
\text { \& surface is S }\end{array}$ & Null & Null & Very low & Low \\
\hline $\begin{array}{c}\text { Resistivity is L } \\
\text { \& surface is S }\end{array}$ & Null & Very low & Low & Medium \\
\hline $\begin{array}{c}\text { Resistivity is H } \\
\text { \& surface is M }\end{array}$ & Very low & Low & Low & Medium \\
\hline $\begin{array}{c}\text { Resistivity is L } \\
\text { \& surface is M }\end{array}$ & Very low & Low & Medium & High \\
\hline $\begin{array}{c}\text { Resistivity is H } \\
\text { \& surface is L }\end{array}$ & Low & Medium & Medium & High \\
\hline $\begin{array}{c}\text { Resistivity is L } \\
\text { \& surface is L }\end{array}$ & Low & Medium & High & Very high \\
\hline
\end{tabular}

Table 1: Example of risk level determination of corrosion under a disbanded shielding coating for pipeline with bituminous coating according to four input variables. For Diameter: $\mathrm{S}=$ small, $\mathrm{L}=$ large; for Resistivity: $\mathrm{L}=$ low, $\mathrm{H}=$ high; for surface: $\mathrm{S}=$ small, $\mathrm{M}=$ medium, $\mathrm{L}=$ large

when the diameter of the pipe is large, the pipe is old (more than 50 years old), the resistivity is low and the estimated defect surface is large.

On the one hand, the number of possible items of qualitative variables is given by the specifications of the database system. They are often described by two or four terms, but sometimes by many more like in the case of the coating type. We often had to use the same granularity to build the fuzzy rules. Sometimes, we were able to group some items to build fuzzy sets, like the one shown in figure 3 telling which coating type is sensitive to stray currents.

On the other hand, quantitative variables description is more flexible because of their continuous domain. We often restrict their description to two or three fuzzy sets due to the number of combinations to consider, like in the case of the estimated defect surface (see figure 4). Indeed, the number of fuzzy rules grows exponentially according to the number of inputs, and it becomes difficult to tell if a parameter is more important than another for each corrosion risk. We ended the modeling with a little more than 300 rules for assessing 9 indicators.

\subsection{Visual tool for case assessment}

A GUI (figure 5) has been designed for the end-users according to their specifications. It is dedicated to the analysis and priorization of DCVG defects. It embeds the inference engine that applies fuzzy rules onto all DCVG defects loaded for analysis. It offers a synthetic view of them with 9 visual indicators. Seven of them stand for an assessed deterioration risk. The two last ones consider an alert about the history of the pipe and its surroundings and the severity level of the consequences of a potential pipeline failure. The list of DCVG defects can be sorted by these scores so that the most critical cases stand out in 


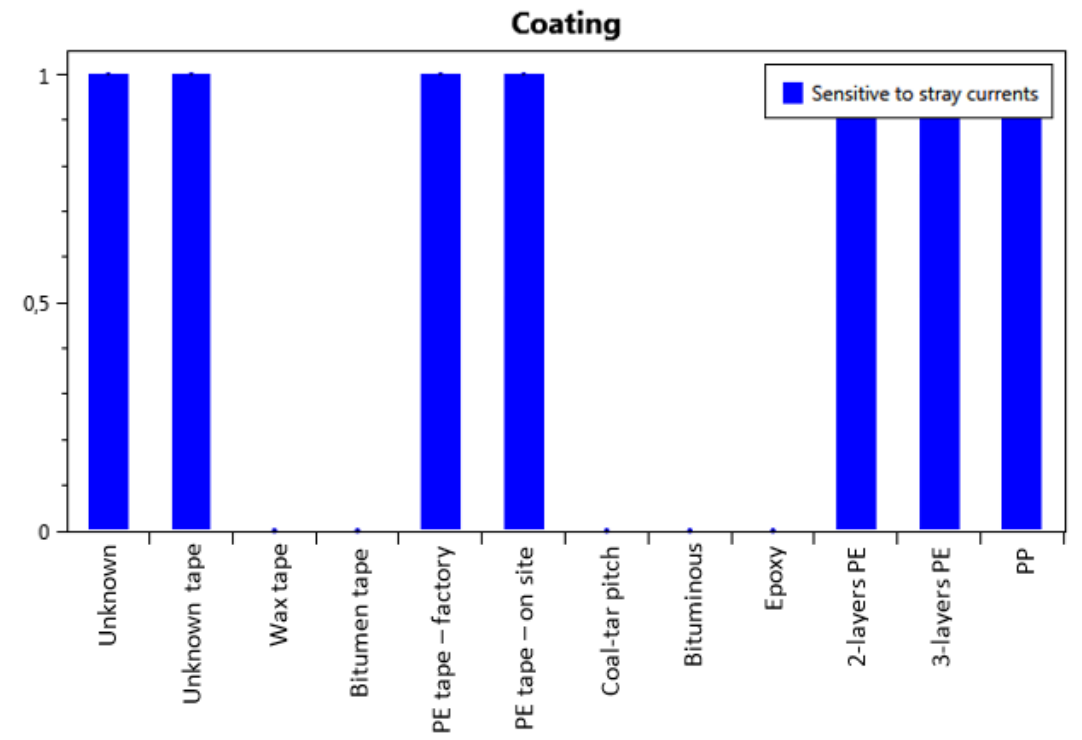

Figure 3: Coating types that are sensitive to stray currents

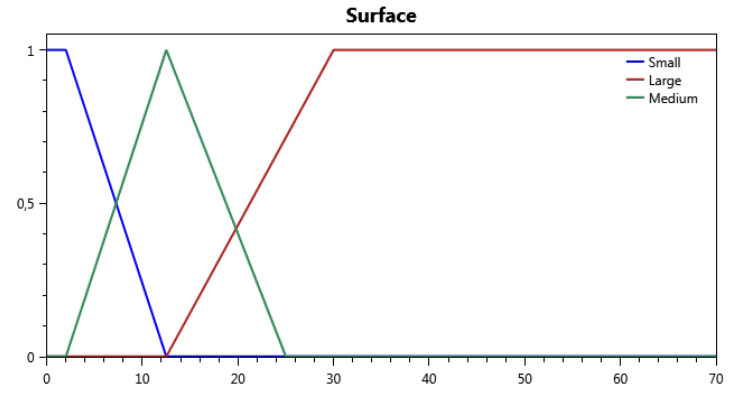

Figure 4: Fuzzy sets defined for the estimated defect surface 


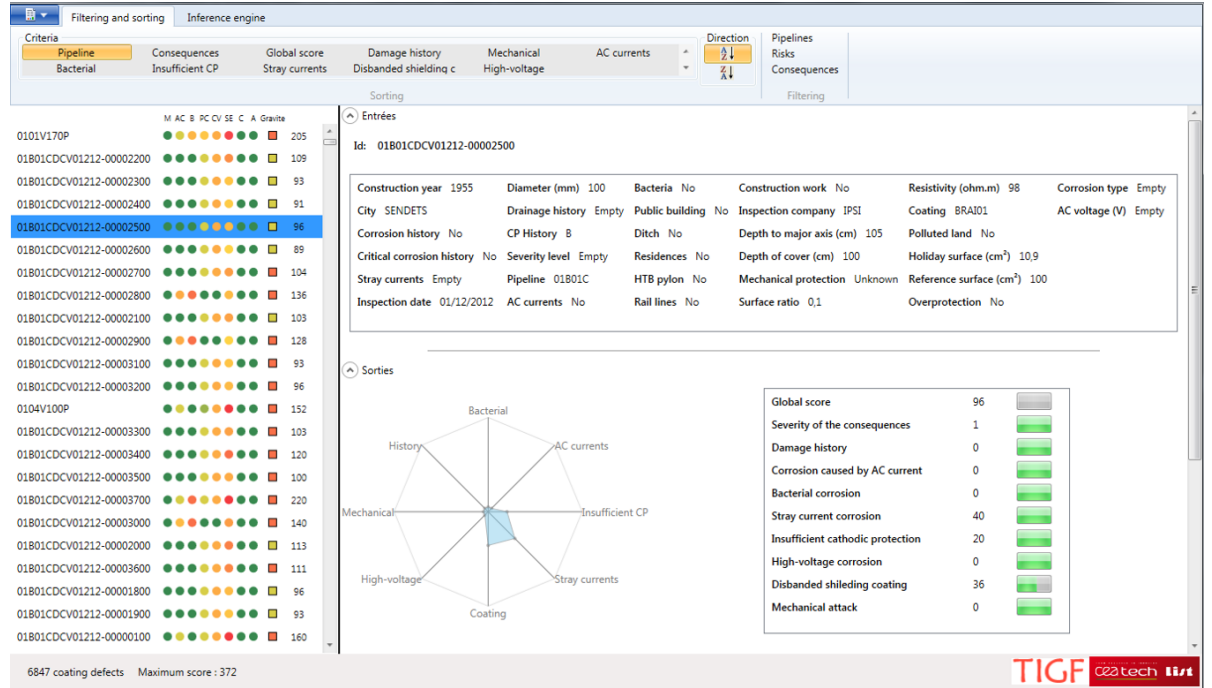

Figure 5: Dedicated user interface with a synthetic view of all the DCVG defects (left part), and a detailed view of a particular defect with its parameter values and the inferred corrosion risks (right part).

a straightforward manner. When a particular defect is selected, its parameter values, the defuzzified values of each indicator and associated activated rules are displayed in specific areas. It is worth mentioning that this feature greatly simplifies feedback integration. Moreover, it improves DSS comprehension and adoption.

\section{Results}

The DSS helps human experts at TIGF to identify and sort the most critical coating defects obtained by a DCVG survey. Before having a field experience return, the evaluation of the DSS results is based on the previous DCVG surveys. Assuming that a threshold decision rule is applied on the higher risk of each coating defect, DSS performance can be assessed like a boolean classification method : either the decision of excavation is correct - that is to say that there is a real metal deterioration of the pipe or its cathodic protection is insufficient - , or the decision is incorrect. The different metrics are then the sensibility (or recall) and the precision defined by the equations 1 and 2 using the notation of the well known confusion matrix (see table 2).

$$
\begin{aligned}
\text { Sensibility } & =T P /(T P+F P) \\
\text { Precision } & =T P /(T P+F N)
\end{aligned}
$$




\begin{tabular}{|c|c|c|c|}
\hline & \multicolumn{2}{|c|}{ System decision } \\
\hline & & Positive & Negative \\
\hline \multirow{2}{*}{ Actual value } & Real defect & True Positive (TP) & False Negative (FN) \\
\hline & No defect & False Positive (FP) & True Negative (TN) \\
\hline
\end{tabular}

Table 2: Confusion matrix

\begin{tabular}{|l|c|c|c|}
\hline Metrics Threshold & $\mathbf{T}=\mathbf{0}$ & $\mathbf{T}=\mathbf{7 0}$ & $\mathbf{T}=\mathbf{8 9}$ \\
\hline Precision & $20 \%$ & $35 \%$ & $73 \%$ \\
\hline Sensibility & $100 \%$ & $55 \%$ & $34 \%$ \\
\hline
\end{tabular}

Table 3: Results according to a threshold $\mathrm{T}$

Applying a null threshold on the highest risk of each defect is the situation of reference, before having the DSS. In that case, only $20 \%$ of excavations are correctly realized (65 cases on 330 excavations). When we use a high threshold $(T=70)$ on the risks estimated by the DSS, $35 \%$ of the excavated pipes would have really been damaged. A little bit more than half of actual defects are retrieved (36 on 65$)$. With a higher threshold $(T=89 \%)$, the selection is even more severe. The precision raises up to $73 \%$, that is to say nearly three excavations out of four would have been decided correctly. However, the rate of actual defects retrieved is only of $34 \%$ (24 cases on 65 ). The goal of the DSS is to help the end-user to better decide which pipe should be excavated. Thus, the goal is to improve the precision of the realized excavations (more actual defects for pipes that are excavated).

\section{Conclusion}

In this paper, we have presented a decision-making tool which has been designed for TIGF, an operator of pipeline network. The goal is to help experts to decide which pipeline section to excavate. The proposed solution relies on a fuzzy expert system which takes the decision regarding 26 criteria and processes more than 300 rules to assess the risk of 7 deterioration types, and two factors indicating if there was an historical damage of the same type and the gravity of the consequences of such a deterioration. The software gives the clues of the decision with charts and the activated rule list, which allows human experts to make the final decision from this explanation of the ranking. Moreover, the software is able to automatically generates reports.

Fuzzy logic is used here to take into account the vagueness of the knowledge of human experts, gathered by iterative interviews: the rules are close to natural language thanks to the linguistic variables and the fuzzy sets avoid using crisp thresholds which have low sense for an experience-based knowledge. Moreover, the ground measures are performed by different workers with different tools and thus come with measurement uncertainty which are easily handled with fuzzy logic.

The results show an improvement of the decision thanks to the tool while it 
is only a formalization of the knowledge of experts at TIGF: up to $73 \%$ of the decisions are accurate and reveal a real defect.

Contrary to other methods, this approach is adaptable to other pipeline network operators: since it relies on a fuzzy expert system, the rule base can be easily adapted to another region of the world if human experts are available to share their knowledge.

The perspectives of this work consist in improving the tool to make it capable of taking advantage of past results to parameterize the rule base and learn new rules.

\section{References}

[1] Athanasopoulos, G., Riba, C.R., Athanasopoulou, C.: A decision support system for coating selection based on fuzzy logic and multi-criteria decision making. Expert Systems with Applications 36(8), 10848 - 10853 (2009)

[2] Bouchon-Meunier, B., Laurent, A., Lesot, M.J., Rifqi, M.: Strengthening fuzzy gradual rules through "all the more" clauses. In: IEEE International Conference on Fuzzy Systems (FUZZ-IEEE). pp. 2940-2946. Barcelona, Spain (2010)

[3] Brito, A., de Almeida, A.: Multi-attribute risk assessment for risk ranking of natural gas pipelines. Reliability Engineering \& System Safety 94(2), 187 -198 (2009)

[4] Cagno, E., Caron, F., Mancini, M., Ruggeri, F.: Using AHP in determining the prior distributions on gas pipeline failures in a robust bayesian approach. Reliability Engineering \& System Safety 67, 275-284 (2000)

[5] Caleyo, F., Valor, A., Alfonso, L., Vidal, J., Perez-Baruch, E., Hallen, J.: Bayesian analysis of external corrosion data of non-piggable underground pipelines. Corrosion Science 90(Supplement C), 33 - 45 (2015)

[6] Cole, I., Marney, D.: The science of pipe corrosion: A review of the literature on the corrosion of ferrous metals in soils. Corrosion Science 56(Supplement C), 5 - 16 (2012)

[7] El-Abbasy, M., Senouci, A., Zayed, T., Parvizsedghy, L., Mirahadi, F.: Unpiggable oil and gas pipeline condition forecasting models. Journal of Performance of Constructed Facilities 30 (2014)

[8] Kishawy, H.A., Gabbar, H.A.: Review of pipeline integrity management practices. International Journal of Pressure Vessels and Piping 87(7), 373 $-380(2010)$

[9] Li, S.X., Yu, S.R., Zeng, H.L., Li, J.H., Liang, R.: Predicting corrosion remaining life of underground pipelines with a mechanically-based probabilistic model. Journal of Petroleum Science and Engineering 65(3), 162 166 (2009) 
[10] Markowski, A.S., Mannan, M.S.: Fuzzy logic for piping risk assessment (pfLOPA). Journal of Loss Prevention in the Process Industries 22(6), 921 -927 (2009)

[11] Martins, A.G., Nicholson, E.: A fuzzy logic model designed for quantitative risk analysis based on ECDA data. In: CORROSION. Dallas (2015)

[12] Poli, J.P., Laurent, J.P.: Touch interface for guided authoring of expert systems rules. In: IEEE International Conference on Fuzzy Systems (FUZZIEEE). pp. 1781-1788 (2016)

[13] Torra, V.: A review of the construction of hierarchical fuzzy systems. International journal of intelligent systems 17(5), 531-543 (2002)

[14] Yang, Y., Khan, F., Thodi, P., Abbassi, R.: Corrosion induced failure analysis of subsea pipelines. Reliability Engineering \& System Safety 159(Supplement C), $214-222$ (2017)

[15] You, Q., Fan, J., Zhu, W., Bai, Y.: Risk assessment of urban gas pipeline network based on fuzzy multi-attribute method. Journal of Applied Sciences 14, 955-959 (2014) 